\title{
Toward a Better Understanding of the Relationship between Childhood Trauma and Psychiatric Disorders: Measurement and Impact on Addictive Behaviors
}

\author{
Adriano Schimmenti ${ }^{\boxplus}$ and Antonia Bifulco ${ }^{2}$ \\ 1'Department of Human and Social Sciences, UKE-Kore University of Enna, Enna, Italy \\ ${ }^{2}$ Department of Psychology, Middlesex University of London, London, UK
}

Craparo, Ardino, Gori and Caretti recently published an interesting brief report in Psychiatry Investigation concerning the role of childhood trauma, dissociation, and alexithymia in alcohol dependence. ${ }^{1}$ In their exploratory study, Craparo and colleagues found that people suffering from alcohol use disorder were exposed to a greater number of traumatic events in their childhood (sexual, physical and emotional abuse), and showed more dissociative experiences and higher levels of alexithymia, compared to a matched control group. Another interesting finding of the study concerned the negative correlation between the age of the first relational trauma and the severity of dissociative symptoms.

This is consistent with a wealth of existing research emphasizing that adverse childhood experiences can alter the affect regulation circuits (alexithymia) and the possibility of integrating mental, somatic, and relational experiences (dissociation), thus constituting a risk-factor for the development of addictive disorders, including the so-called behavioral addictions such as pathological gambling ${ }^{2}$ and Internet addiction. ${ }^{3}$ The study by Craparo and colleagues provides additional evidence in a growing field for the influence exerted by negative childhood environment and relationships in the development of addictive disorders. As the literature indicates, people suffering from addictive disorders may in fact use substances or excessive behaviors as an external regulator and a self medication for their dysregulated feelings and painful states of mind. This then supports a psychopathological model according to which

Received: September 4, 2014 Revised: November 2, 2014

Accepted: November 11, 2014 Available online: July 6, 2015

$\triangle$ Correspondence: Adriano Schimmenti, $\mathrm{PhD}$

Department of Human and Social Sciences, UKE -Kore University of Enna, Cittadella Universitaria, CAP 94100, R.14, Enna, Italy

Tel: +39-3286267944, Fax: +39-0935536943

E-mail: adriano.schimmenti@unikore.it

(a) This is an Open Access article distributed under the terms of the Creative Commons Attribution Non-Commercial License (http://creativecommons.org/licenses/bync/3.0) which permits unrestricted non-commercial use, distribution, and reproduction in any medium, provided the original work is properly cited. the earlier and more frequent the exposure to abuse for an individual, the higher the likelihood of deviation from normal development. In fact, both theoretical and empirical research states that early and frequent negative experiences in childhood, especially in the context of attachment relationships, can foster the onset of a developmental trauma disorder, ${ }^{4}$ which entails significant alterations in different domains of psychological functioning, including attachment, biology, affect regulation, dissociation, behavioral control, cognition and self-concept. $^{5}$

Therefore, research findings are consistent in supporting developmental models for psychiatric disorders, whereby childhood neglect or abuse is an important causal factor, increasingly for a range of disorders. However, it is now necessary for research on the developmentally traumatic roots of addictive behaviors to make a quantum leap, in order to provide more detailed patterns of associations between childhood experiences and addictive behaviors in a search for more specific models. In doing this, a valid, reliable, comprehensive, and detailed assessment of child maltreatment becomes essential for increasing our understanding of the origins of addictive behaviors in addition to other forms of psychopathology. In fact, given potential methodological issues around retrospective measurement, accurate recall, and willingness to report sensitive or stigmatizing personal information, the choice of a suitable measure of childhood maltreatment is a critical issue for both research and clinical purposes.

In this sense, the Childhood Experience of Care and Abuse (CECA) interview ${ }^{6}$ is unique as the first, most comprehensive, well tested measure of child maltreatment, with published reliability and validity (including a sibling corroboration study), ${ }^{7}$ and it has been used in patient and community samples internationally. ${ }^{8-10}$ The only other similar interview is the Childhood Trauma Interview, ${ }^{11}$ which has a narrower range, less validational testing and less international use, but which has recently 
been adapted to follow the CECA in utilizing a wider range of indices for analysis. ${ }^{12}$ The CECA is considered the gold standard for the retrospective assessment of both trauma and lack of care experienced during childhood, due to its good psychometric properties, its careful, detailed, and behaviorallybased questioning, and wide range of experiences covered. In the 20 years since its initial publication, there are more than 100 studies with this measure that have identified specific patterns of psychopathological development. For example, studies with the CECA show that emotional neglect in childhood is specifically associated with the development of anxiety disorders ${ }^{13}$ and depressive disorders, ${ }^{14}$ and that role-reversal ${ }^{15}$ (i.e., parentification) and physical abuse ${ }^{16}$ are associated with the development of suicidal and self-injurious behaviors. Another recent study with the CECA showed that witnessing violence in the household is specifically associated with the development of substance use disorder among late adolescents. ${ }^{17}$ Other analyses point to differentiation of disorder according to parent involved with maternal neglect/abuse relating to internalizing disorders (e.g., anxiety, depression) and paternal neglect/abuse to externalizing disorder (e.g., conduct disorder, substance use disorder, antisocial personality disorder). ${ }^{17-19}$ On the basis of these and other findings, we believe that the use of the CECA measure and using an intensive interview approach can dramatically improve the quality of research data on the relationship between child abuse and addictive behaviors, as well as improving the understanding of the relationship between childhood maltreatment and psychopathology more generally. This is not to underrate the usefulness of self-report screening tools for certain types of study, in particular for screening studies-the CECA.Q, ${ }^{20}$ a selfreport measure based on the CECA interview, has proved robust in showing early experience influencing later disorder, and dose-response effects, but without the range of the interview measure to develop specificity of models, and the safeguards imposed in the rating scheme on accurate reporting.

It has been illuminating in the 20 years since the CECA was first published, to see its initial success in the prediction of depression being widely replicated but for a range of disorders. The measure has proved robust over time and across culture, and using the full range of the CECA measurement potential will allow for ever more specific models of disorder in relation to type of childhood maltreatment, by particular perpetrators and at specific ages. We believe that this kind of retrospective assessment for child maltreatment can make all the difference for developing consistent psychopathological model to inform clinical practice with individuals who have been traumatized in childhood.

\section{REFERENCES}

1. Craparo G, Ardino V, Gori A, Caretti V. The relationships between ear- ly trauma, dissociation, and alexithymia in alcohol addiction. Psychiatry Investig 2014;11:330-335.

2. Scherrer JF, Xian H, Kapp JM, Waterman B, Shah KR, Volberg R, et al. Association between exposure to childhood and lifetime traumatic events and lifetime pathological gambling in a twin cohort. J Nerv Ment Dis 2007;195:72-78.

3. Schimmenti A, Passanisi A, Gervasi AM, Manzella S, Famà FI. Insecure attachment attitudes in the onset of problematic Internet use among late adolescents. Child Psychiatry Hum Dev 2014;45:588-595.

4. van der Kolk BA. Developmental trauma disorder: toward a rational diagnosis for children with complex trauma histories. Psychiatr Ann 2005;35:401-408.

5. Cook A, Spinazzola J, Ford J, Lanktree C, Blaustein M, Cloitre M, et al. Complex trauma in children and adolescents. Psychiatr Ann 2005; 35:390-398.

6. Bifulco A, Brown GW, Harris TO. Childhood Experience of Care and Abuse (CECA): a retrospective interview measure. J Child Psychol Psychiatry 1994;35:1419-1435.

7. Bifulco A, Brown GW, Lillie A, Jarvis J. Memories of childhood neglect and abuse: corroboration in a series of sisters. J Child Psychol Psychiatry 1997;38:365-374.

8. Giannone F, Schimmenti A, Caretti V, Chiarenza A, Ferraro A, Guarino $S$, et al. Validity, reliability, and psychometric properties of the Italian translation of the CECA interview. Psichiatria e Psicoterapia 2011;30:3-21.

9. Kaess M, Parzer P, Mattern M, Resch F, Bifulco A, Brunner R. Childhood Experiences of Care and Abuse (CECA) - validation of the German version of the questionnaire and interview, and results of an investigation of correlations between adverse childhood experiences and suicidal behaviour. Z Kinder Jug-Psych 2011;39:243-252.

10. Harkness KL, Wildes JE. Childhood adversity and anxiety versus dysthymia co-morbidity in major depression. Psychol Med 2002;32:12391249.

11. Fink LA, Bernstein D, Handelsman L, Foote J, Lovejoy M. Initial reliability and validity of the childhood trauma interview: a new multidimensional measure of childhood interpersonal trauma. Am J Psychiatry 1995;152:1329-1335.

12. Vrshek-Schallhorn S, Wolitzky-Taylor K, Doane LD, Epstein A, Sumner JA, Mineka S, et al. Validating new summary indices for the Childhood Trauma Interview: associations with first onsets of major depressive disorder and anxiety disorders. Psychol Asses 2014;26:730-740.

13. Schimmenti A, Bifulco A. Linking lack of care in childhood to anxiety disorders in emerging adulthood: the role of attachment styles. Child Adolesc Ment Health 2013;20:41-48.

14. Harkness K, Monroe SM. Childhood adversity and the endogenous versus nonendogenous distinction in women with major depression. Am J Psychiatry 2002;159:387-393.

15. Bifulco A, Schimmenti A, Moran P, Jacobs C, Bunn A, Rusu AC. Problem parental care and teenage deliberate self-harm in young community adults. Bull Menninger Clin 2014;78:95-114.

16. Ystgaard M, Hestetun I, Loeb M, Mehlum L. Is there a specific relationship between childhood sexual and physical abuse and repeated suicidal behavior? Child Abuse Negl 2004;28:863-875.

17. Bifulco A, Schimmenti A, Jacobs C, Bunn A, Rusu AC. Risk factors and psychological outcomes of bullying victimization: a community-based study. Child Indic Res 2014;7:633-648.

18. Bifulco A, Thomas G. Understanding Adult Attachment in Family Relationships: Research, Assessment and Intervention. London, UK: Routledge; 2012.

19. Giovagnoli O, Ducro C, Pham TH, Woitchik P. Impact of familial adversities during childhood on the onset of psychopathic personality. Ann Med Psychol 2013;171:509-511.

20. Bifulco A, Bernazzani O, Moran PM, Jacobs C. The childhood experience of care and abuse questionnaire (CECA.Q): validation in a community series. Br J Clin Psychol 2005;44:563-581. 